cross boundary flow funding for regional specialties would remove from the regional health authority the control over the volume of work that it has at present. Therefore, a proposal that is managerially sound and operates in the private sector and in health services overseas is politically unacceptable in Britain.

David S GRIMES

Postgraduate Medical Centre,

Lancashire BB2 3LR

\section{Treating Paget's disease}

SIR,-DD D A Heath's article on the treatment of Paget's disease shows a worrying degree of complacency over this chronic condition (25 April, p 1048). The argument that most patients with this disease do not need to be treated is an old and cherished one, but we believe that Dr Heath has overstated it.

As we have gained experience with the use of intravenous aminohydroxypropylidene diphosphonate in the form of CGP 23339 (not yet commercially available), ${ }^{1}$ which is clearly much safer and more effective than oral etidronate, our threshold for treatment has fallen progressively. We have now treated over 100 patients, and a striking feature is that many with rather vague bone pain have general ill health and malaise, which also shows sustained improvement after effective treatment. It seems possible that improved and fairly short treatment regimens with aminohydroxypropylidene diphosphonate will cure the disease without running the major risk of osteomalacia and bone fracture, which are seen with etidronate; the evidence so far is that the drug is extremely safe in both the short and the long term. If this is borne out by further experience the threshold for treatment will fall even lower. Treatment will become routine on diagnosis of Paget's disease, especially in younger patients, who obviously have a long time ahead for the disease to progress. With regard to many of the complications, it is obvious that cure will be the best means of prevention. Leaving it to the drug companies to set the pace for developing and applying new treatments for Paget's disease is in our opinion to shirk our responsibilities.

\section{DAVID C ANDERSON}

J A CANTRILL

Department of Medicine,

University of Manchester,

Salford M6 8HD

1 Cantrill JA, Buckler HM, Anderson DC. Low dose intravenous 3-amino-1-hydroxypropylidene -1,1- bisphosphonate (APD) for the treatment of Paget's disease. Ann Rheum Dis 1986;45: for the

AUTHOR'S REPLY,-Professor Anderson's work on his first 20 cases treated with intravenous aminohydroxypropylidene diphosphonate was known to me when I wrote my review because he was good enough to let me have a preprint of his paper. The essence of his argument is that treatment with aminohydroxypropylidene diphosphonate is safer than oral etidronate and more effective and improves general ill health and malaise. He also seems to be suggesting that treatment may cure the disease.

In my review I discussed the safety of etidronate and, when used at low doses, it does not seem to carry a risk of clinical osteomalacia or increased fracture rate. Aminohydroxypropylidene diphosphonate is undoubtedly more effective than etidronate in controlling the biochemical markers of the disease, but it remains to be shown that this carries any advantage over mere relief of pain. Informed judgment on the claim to improve ill health is impossible in the absence of proper randomised controlled comparisons. Placebo responses are recorded in as many as $40 \%$ of patients with Paget's disease.

The results in the first 20 patients treated were submitted for publication in June 1986. Presumably the additional 80 patients have therefore been treated in the past 12 months. It seems unwise to talk in terms of cure with such limited follow up.

Whatever Professor Anderson and $\mathrm{Mr}$ Cantrill may say, despite the fact that for over eight years favourable reports of aminohydroxypropylidene diphosphonate have appeared, the drug is not available for general prescribing. Possible reasons for this were given in my review. The drug may be obtained on a named patient basis, but the widespread use of the drug in this way would be wrong as it would be bypassing the mechanism for regulating the use of new drugs. Named patient use should be reserved for patients who cannot be managed with existing treatment.

D A Heath

University Department of Medicine,

Queen Elizabeth Hospital,

Birmingham B15 2TH

\section{Bone mineral density in Addison's disease}

SIR,-Dr J P Devogelaer and colleagues claim to have produced evidence for an effect of adrenal androgens on bone mass (28 March, $p$ 798). This is a subject that exercised the Leeds group for some time. Our description of reduced adrenal sex hormone concentrations in subjects with early or accelerated postmenopausal osteoporosis and the decline in these hormones in women at about age 60 led us to resurrect the idea of the adrenopause and propose that the postmenopausal woman with accelerated osteoporosis had undergone an early adrenopause. ${ }^{12}$ The idea of the adrenopause and that adrenal androgens are important in bone metabolism was, I believe, originally suggested by Albright and Reifenstein in 1948. ${ }^{3}$ As androgens serve as precursors for oestrogens, however, it has never been clear whether their effect, if any, is direct or indirect through oestrogen production.

The report by Dr Devogelaer and coworkers does not seem to resolve this issue. The rate of calcium loss and the postmenopausal oestrone concentration are known to be related, ${ }^{46}$ and the reduced oestrone concentration may therefore account for the lower bone mass in the postmenopausal patients with Addison's disease. The oestrone concentrations reported in their study seem, if anything, rather high.

The preservation of bone in the men does not necessarily mean that the androgens are responsible. Men have an oestrogen concentration above that of postmenopausal women, and it has been suggested that this may contribute to the preservation of bone in aging men. ${ }^{4} \mathrm{Dr}$ Devogelaer and colleagues do not report the hormone concentrations in the men with Addison's disease, but it is unlikely that the oestradiol concentrations would be much affected.

If we are looking for an effect of androgens, independent of any action through conversion to oestrogens, then we should look at androgen deficient, oestrogen replete subjects. The ideal model would be postmenopausal patients with Addison's disease receiving oestrogen replacement. The group in the paper by Dr Devogelaer and coworkers who come closest to this are the premenopausal women, who are oestrogen replete but have lost between a half and two thirds of their androstenedione and testosterone and practically all of their dehydroepiandrosterone. These patients, however, have not lost bone.

The relative balance between sex steroids and glucocorticosteroids may be important. Premeno- pausal women seem to be fairly well protected against the effects of glucocorticoid treatment. ${ }^{8}$ It may well be that high glucocorticosteroid concentrations may be counteracted by high oestrogen concentrations. On the other hand, physiological replacement doses of glucocorticoids might do harm in the presence of extreme sex hormone deprivation-as in postmenopausal patients with Addison's disease.

The precise physiological role of the adrenal sex hormones in older life seems unclear. Why they should be secreted in response to adrenocorticotrophic hormone is also unclear, but it would make an attractive hypothesis to propose that androgen secretion, occurring hand in hand with the physiological secretion of glucocorticoids, protects some parts of the body, such as bone, from the catabolic effects of glucocorticoids: If so, however, whether the effect is direct or through the secondary oestrogen production remains unresolved.

\section{University of Western Ontario,}

RICHARD G CRILLY Ontario N6C 5J1

1 Crilly RG, Marshall DH, Nordin BEC. Adrenal androgens in postmenopausal osteoporosis. In: Genazzani AR, Thijssen JHH, Siiteri PK, eds. Adrenal androgens. New York: Raven Press, 1980:241-58.

2 Crilly RG, Marshall DH, Nordin BEC. Effect of age on plasm androstenedione concentration in oophorectomised women. Clin Endocrinol (Oxf 1979;10:199-201.

Albright F, Reifenstein EC. The parathyroid glands and metabolic bone disease. Baltimore: William and Wilkins, 1948

Crilly RG, Francis RM, Nordin BEC. Steroid hormones, agein and bone. $\mathcal{J}$ Clin Endocrinol Metab 1981;10:115-39.

Lindsay R, Coutts JRT, Hart DM. The effect of endogenous oestrogens on plasma and urinary calcium and phosphate in oophorectomised women. $\mathcal{J}$ Clin Endocrinol Metab 1977; 6 : 87-93.

6 Frumar AM, Meldrum DR, Geola F, et al. Relationship of fasting urinary calcium to circulating oestrogen and body weight in postmenausal 7 Clin Endocrinol Merab 1980; 50 : 70-5.

Crilly R, Marshall DH, Cawood M, Nordin BEC. Hormona status in normal, osteoporotic and corticosteroid-treated postmenopausal women. $\mathcal{J} R$ Soc Med 1978;71:733-6.

Crilly RG. Corticosteroid induced osteoporosis. Belfast: Queen' University Medical School, 1981. (Thesis.)

\section{HIV testing and differential diagnosis}

SIR,-Among the excellent points made in the $\mathrm{ABC}$ of AIDS, one recommendation sets my teeth on edge. This is the unqualified insistence that the diagnosis should be discussed with the patien before testing for human immunodeficiency virus (HIV).

In some contexts this is clearly excellent advice: in voluntary screening programmes, in genitourin ary medicine clinics, when the patient is known to be in a high risk group, and when the patien expresses concern about the possibility. However we can expect increasingly to see the condition presenting in general practice and in clinics of general and respiratory medicine, neurology, gastroenterology, and dermatology. The propor tion of heterosexuals affected is likely to increase and patients may not be aware of exposure to the virus. Previously unsuspected groups may prove to be at risk. In any such individual patient HIV infection may be only one possibility, perhaps a remote one, in a long list of differential diagnoses, but it needs to be excluded or confirmed in the patient's interest and in the wider interest of following the progress of the epidemic.

Many of us would be deterred from ordering HIV antibody testing if we felt that standard practice entailed facing the patient with the possibility of AIDS in such circumstances. Afte all, we do not discuss the implications of leukaemia before ordering a white cell count or of bronchia carcinoma when ordering a chest radiograph. Even after receiving a positive or suspicious result we are likely to seek confirmation, either by repeating the 
test or through collateral evidence, especially as human errors can so easily arise in the chain of sending specimens and reports even in the best run hospitals. We would raise the possibility of such serious disease with an unsuspecting patient only after collecting evidence and reaching a degree of certainty.

I suggest that precounselling should be omitted when HIV testing is to be done as part of differential diagnosis in a patient who is not overtly concerned about HIV infection. If the result is negative the need for repeat testing will be determined by other findings and subsequent progress. If it is positive the clinician may choose not to reveal the result immediately but rather to raise the possibility of HIV infection with the patient and proceed to a confirmatory HIV antibody test.

Doctors are paid not to obey rules but to exercise judgment in the patients' best interests. These interests are not served by raising the spectre of AIDS (or of any other serious disease) prematurely and unnecessarily.

J G DiCKINSON

Queen Elizabeth Military Hospital,

Woolwich SE18 4QH

\section{AIDS: When to test}

SIR,-Your series on the acquired immune deficiency syndrome (AIDS) is both timely and helpful. It has, however, raised at least one major doubt in my mind: when to investigate.

AIDS is a polymorphic disease, as the articles show. The differential diagnosis of lymphadenopathy (which is an early feature in some patients) includes the equally serious disease leukaemia. Leukaemia is often treatable and must therefore be identified as early as possible. In general practice the order in which investigations are carried out is related to the likelihood of the test being helpful, its cause, and its invasiveness. All other things being equal, a blood film is always done before a bone marrow biopsy.

With AIDS we are told that a blood test for the presence of antibodies should not be done before the patient has been counselled and fully understands the effects of having such a test. I am aware of the limitations of the human immunodeficiency virus (HIV) antibody test, but, to some extent, similar limitations apply to many other investigations but do not prevent us from performing the tests. Nor do we always tell the patient every possible outcome of an investigation. Indeed, we ourselves are sometimes surprised when an investigation shows a possible lesion that we had not previously considered. Why should a test for HIV antibody be different?

STUART CARNE

Grove Health Centre

London W12 8EJ

\section{Simple thyroid cyst: cause of acute bilateral} recurrent laryngeal nerve palsy

SIR,-We agree with Messrs J S Gani and J M Morrison (2 May, p 1129) that it is difficult to explain the rapid onset and sudden relief of the palsy in their patient. We think, however, that impairment of the microcirculation to both nerves is an unlikely cause.

Interruption of the microcirculation to a nerve for a short period of 20 to 30 minutes results in a palsy with no recognisable pathological lesion, and on re-establishing the blood flow there is an almos immediate reversal. ${ }^{1}$ Longer lasting ischaemia results in demyelination with a far longer recovery period. In the reported case the microcirculation to the recurrent laryngeal nerves was probably interrupted for more than 30 minutes, making immediate recovery, when the pressure was relaxed, extremely unlikely.

In January this year we admitted a 41 year old man who had had a goitre for many years and who gave a three month history of hoarseness. Indirect laryngoscopy showed a paralysed left vocal cord, but at operation the left recurrent laryngeal nerve was noted to be in a normal anatomical position and not stretched over the gland. The left vocal cord remained paralysed in the immediate postoperative period. Histological examination of the gland confirmed the diagnosis of a multinodular goitre. At his second outpatient visit 10 weeks after surgery his voice had improved but the left vocal cord remained paralysed. When he reattended six weeks later he had further improvement with full vocal cord movement.

We believe that this patient's recovery was more typical of the pattern exhibited by patients with ischaemic demyelination injury to the recurrent laryngeal nerve.

A R Quayle

C H TALBoT

Royal Hallamshire Hospital,

Sheffield S10 2JF

1 Ochoa J. Nerve fiber pathology in adult and chronic compression. In: Omer GE, Spinner M, eds. Management of peripheral nerve problems. Philadelphia: Saunders, 1980.

\section{Developing primary health care}

SIR,-Professor Brian Jarman and Mrs Julia Cumberlege (18 April, p 1005) propose a totally organised system of community care on a geographical basis. Their system would work if all general practitioners were state salaried and their premises owned by the state. Commendably, they include the general practitioner in their primary health care team, but it is astonishing that "there is no question of one person being in charge." Really. Consensus management went out with Griffiths, and now he too is looking at community services. Furthermore, there is no word about practice nurses (there could easily be 10000 in the country), yet the authors say, "We support attempts to evaluate different models of care."

It is surely arrogant to say that "The patient's right to freedom of choice of a doctor, however, should be retained, as it adds flexibility to the rigidity of fixed geographically based services." There is more to a patient's freedom of choice than this. A patient has allegiance to a practice, not a community, and the reference point in community care is the practice and not the community care area. Mrs Cumberlege has enhanced the role of the practice nurse enormously. Let us hope that the paper by Professor Jarman and Mrs Cumberlege has a similar paradoxical effect.

D U BLOOR

West Midlands DY6 9JQ

\section{Clinical Algorithms: Irregular vaginal bleeding}

SIR,-It is a commonly held belief that carcinoma of the cervix is always treated by hysterectomy and that some sort of adjuvant radiotherapy is sometimes given for the sake of completeness. Unfortunately, Professor Geoffrey Chamberlain seems to imply this in his recent article (11 April, p 947). He states: "If the biopsy confirms the presence of stratified squamous cell carcinoma (or, rarely, adenocarcinoma from the canal) the treatment is usually a combination of radical surgery, local irradiation, and deep irradiation," and in the algorithm box he puts "Radical surgery \pm deep $x$ ray treatment."

Though a combined approach is generally accepted for the management of operable carcinoma corpus uteri, there are few.cases of invasive cancer of the cervix (nearly always stage IB or earlier) in which the treatment of choice is not primary radiotherapy with external beam and subsequent intracavitary irradiation. For more advanced stages surgery is not a feasible proposition, and as yet such cases form the bulk of the caseload for invasive cancer of the cervix in most centres.

Postoperative radiotherapy is often requested for patients in whom pelvic lymph node metastases were found at surgery. In fact, very few such patients really benefit much unless the node disease is truly microscopic and can therefore be eradicated by the radiation doses which can then "safely" be given. (The place of aggressive chemotherapy deserves more serious consideration here than it commonly receives.) The opposite approach, when primary radical radiotherapy is followed by planned surgery, has its advocates, though usually for barrel shaped tumours or adenocarcinomas. I am reliably informed by gynaecologist colleagues, however, that hysterectomy after radiotherapy is rarely a radical operation, unless the radiation doses were deliberately kept low.

Planned combinations of radical treatments carry unnecessarily high risks of damage to patients and rarely improve the prognosis for the patients. "Safe" combinations, given in either order of preference, inevitably mean that the efficacy of one of the treatments is compromised; the patient's medical attendants probably gain more in reassurance than the patient gains in prognosis. Obviously, the radiotherapist must attempt what he can for a patient in whom primary surgery is inadequate, just as the gynaecologist rarely objects to attempting salvage surgery in the patient for whom radiotherapy has failed.

My complaint is that so often a radiotherapist has to answer the questions of an anxious patient, or her husband, who has been told by junior gynaecological staff, the family doctor, or a nurse to expect major surgery-whatever the disease stage - and who then understandably thinks that radiotherapy is either second best or simply wrong. I realise that Professor Chamberlain could not enter this kind of discussion for the purposes of his algorithm, and I do not wish to open up the silly surgery versus radiotherapy argument. I would suggest, however, that the algorithm box for the treatment of cancer of the cervix should more accurately be labelled "Surgery or radiotherapy."

I J KerBY

South Wales Radiotherapy and Oncology Service,

Velindre Hospital,

Cardiff CF4 7XL

\section{Look after your heart}

SIR,-Although Dr Noel D L Olsen's leading article (2 May, p 1115) contains much good sense, it conspicuously failed to mention promotion of exercise as a priority in coronary prevention. This omission is unfortunate not only because exercise is highly effective in prevention but also because attention to other risk factors has not substantially altered the overall incidence of coronary disease.

Paffenbarger showed lack of exercise to be the best predictor of coronary risk in the community he studied, ${ }^{2}$ and in May's review ${ }^{3}$ physical conditioning was the best form of secondary prevention (Coronary Prevention Group, RSM, London, 1987). Remarkably little exercise is required to reduce coronary risk (30 minutes 